\title{
Quantification of Elemental Distribution in Spherical Core-Shell Nanoparticles Measured by STEM-EDX.
}

\author{
Jacob T. Held ${ }^{1}$, Katharine Hunter ${ }^{2}$, Uwe R. Kortshagen ${ }^{2}$, K. Andre Mkhoyan ${ }^{1}$ \\ ${ }^{1 .}$ Department of Chemical Engineering \& Materials Science, University of Minnesota, Minneapolis, \\ MN. \\ 2. Department of Mechanical Engineering, University of Minnesota, Minneapolis, MN.
}

Quantum dots exhibit many interesting and useful size-dependent optoelectronic properties and are used extensively in fields ranging from optical displays to biological imaging. ${ }^{1,2}$ By adding shell structures to these quantum dots, many properties may be improved, including mitigation of surface effects, reduction in blinking, and control of spacing between deposited particles. ${ }^{3,4}$ Alternatively, core/shell structures can be fabricated to create various types of band alignment between the core and shell materials: one example is type-II confinement, which has been studied systems such as $\mathrm{Ge} / \mathrm{Si}^{5}$ and $\mathrm{CdTe} / \mathrm{CdSe}{ }^{6}$ core/shell particles. The optical properties of these systems are affected by the quality of the interface between the two materials. As such, interfacial broadening between the core and shell is an important parameter to measure and control in these systems. This elemental distribution, while important, has proven to be difficult to accurately assess. ${ }^{7}$ In this study, we use plasma-grown spherical Ge/Si core/shell nanoparticles as a test case for scanning transmission electron microscopy-energy dispersive X-ray (STEM-EDX) analysis of the core/shell elemental distribution.

$\mathrm{Ge} / \mathrm{Si}$ core/shell nanoparticles were grown in a plasma reactor where Ge cores were generated in a $\mathrm{GeCl}_{4} / \mathrm{Ar} / \mathrm{H}_{2}$ plasma. The shells were formed by injecting a $\mathrm{SiH}_{4} / \mathrm{He}$ mixture into the plasma afterglow. The particles were directly deposited onto ultra-thin/holey double carbon grids and transferred under Ar to an FEI-Titan G2 60-300 equipped with a Gatan Enfinium EEL spectrometer and a Super-X EDX system. EDX spectrum images of the particles were acquired at $60 \mathrm{kV}$ with a 25 mrad convergence angle, $200 \mathrm{pA}$ beam current during which no damage to the particles was detectable. The Si and Ge K edges were background-subtracted and integrated, producing spectrum images (Figure 1).

An ellipse was fit to each dataset to determine the centroid of the particles. Only particles for which the major and minor axes were within $<5 \%$ were used in order to support a spherical geometry approximation. The data was then radially averaged around the fit centroid and a 3 pixel moving average was applied to reduce noise. A continuum core/shell model was developed to describe interdiffusion of the core and shell material. This model was projected onto a 2D profile and convoluted with a Gaussian to account for probe spread. The simulation parameters (core and shell radii, interface broadening, and Gaussian spread function) were then fit to the experimental data by minimizing the sum-squared error, generating a spherical concentration map, such as that shown in Figure 2. This approach appears to be a promising technique to quantify interface broadening of core/shell nanostructures with well-defined geometries. [8]

\section{References:}

[1] K. Bourzac. Nature 493 (2013) p. 283.

[2] M. Bruchez, Jr. et al. Science 281 (1998) p. 2013.

[3] Y. Chen, et al. J. Am. Chem. Soc. 130 (2008) p. 5026.

[4] C. P. Byers et al. Sci. Adv. 1 (2015) 
[5] E. L. de Oliveira, et al. J. Phys. Chem. C 116 (2012) p. 4399.

[6] C.-H. Chuang, et al. ACS Nano. 5 (2011) p. 6016.

[7] N. Tschirner, et al. Chem. Mater. 24 (2012) p. 311.

[8] This work was supported primarily by NSF through the University of Minnesota MRSEC under Award Number DMR-1420013.
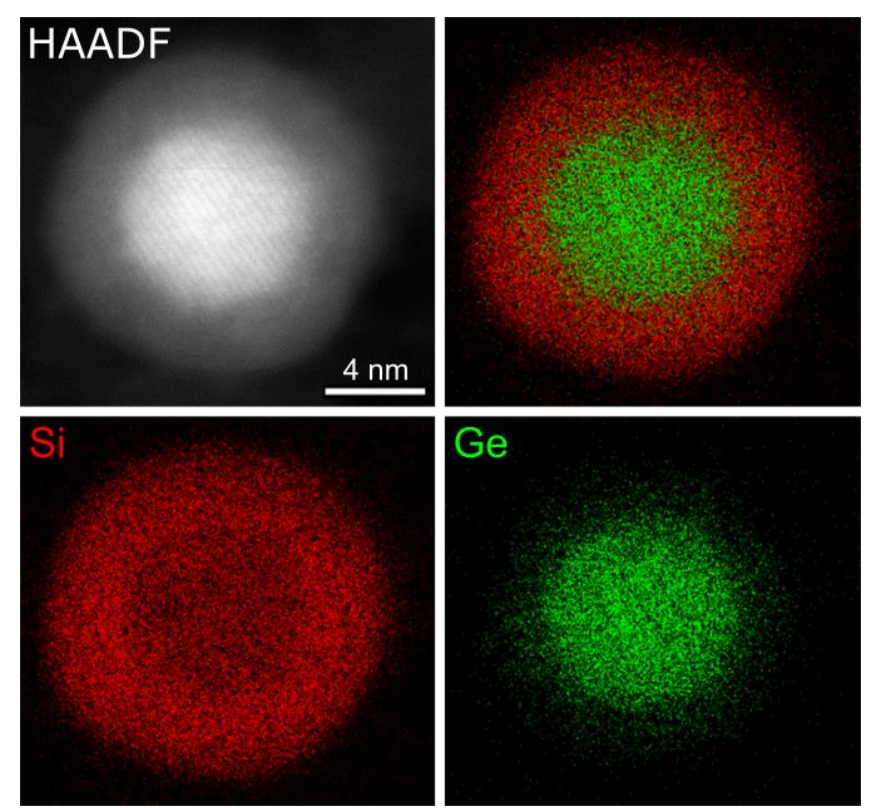

Figure 1. Sample dataset of a spherical $\mathrm{Ge} / \mathrm{Si}$ core/shell nanoparticle including a HAADF image, composite Ge + Si EDX spectrum image, and independent Si and Ge maps. The Si and Ge K edges were background-subtracted and integrated in each pixel. The maps shown have been scaled from 0-255 and a 3-pixel moving average was applied for clarity.
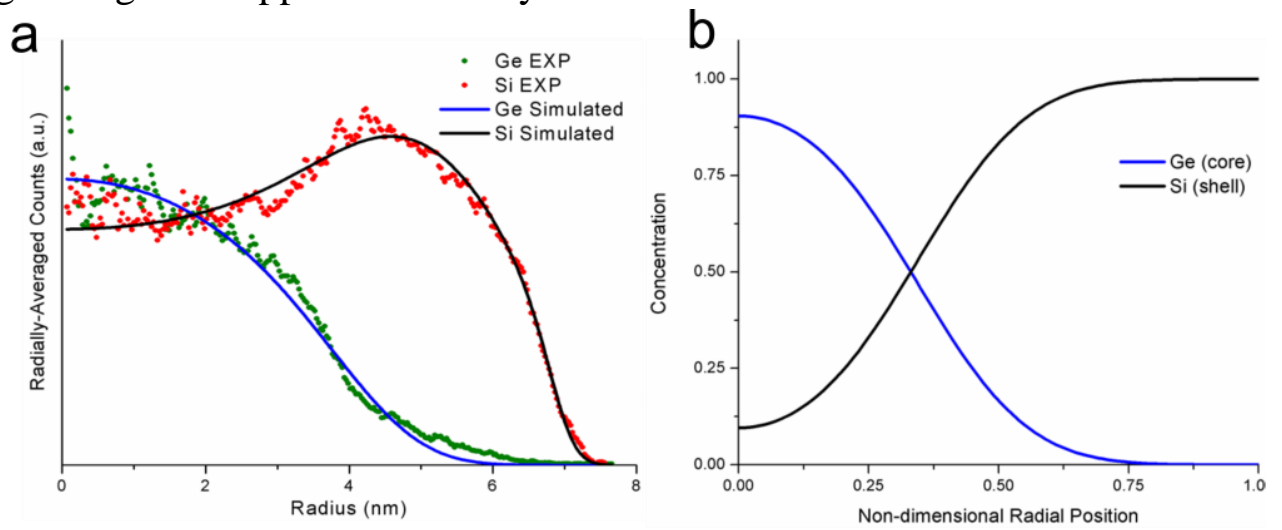

Figure 2. (a) Radially averaged map of Ge and Si EDX counts scaled and plotted alongside the best-fit simulated projection. A 3-pixel moving average was applied to reduce noise. (b) Spherical concentration map of the simulation plotted in (a). 